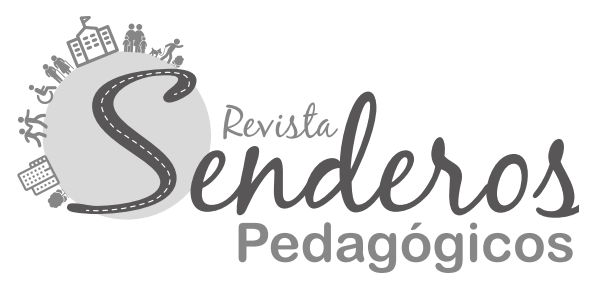

\title{
Narrativas sobre el saber pedagógico, entre el acierto y la adversidad: reflexiones desde la educación rural en el nordeste de Antioquia ${ }^{1}$
}

\author{
Narratives on pedagogical knowledge, between success and \\ adversity: reflections from rural education in the northeast of Antioquia
}

\begin{abstract}
Autores:
Julián Alexis Cataño Duque ${ }^{2}$, Beatriz Elena Cortés Arango 3

Recibido: 08/05/2020

Aprobado: 29/09/2020

1. Artículo de reflexión.

2. Licenciado en Filosofía y Educación Religiosa de la Fundación Universitaria Católica del Norte, Magíster en Neuropsicología y educación de la Universidad Internacional de la Rioja. jualcadu1992@gmail.com

3. Historiadora de la Universidad de Antioquia, Especialista en Políticas Públicas para la Igualdad del Consejo Latinoamericano De Ciencias Sociales (CLACSO). elecor11@gmail.com
\end{abstract}

\section{Resumen:}

La educación en el contexto rural está determinada por situaciones históricas, políticas, sociales, económicas y culturales que, pese a su enorme trascendencia para la población más vulnerable de Colombia, ha sido desde hace mucho tiempo invisibilizada, marginada, empobrecida y distanciada de los discursos académicos e investigativos. Este artículo reflexivo es un acercamiento a las historias de los y las maestras rurales del departamento de Antioquia, a partir de las narrativas autobiográficas realizadas en el año 2018 en el Centro de Pensamiento Pedagógico, buscando visibilizar la labor docente que se desempeña en la ruralidad del nordeste antioqueño, en donde históricamente han emergido problemas a raíz del conflicto armado, la violencia, el abandono del Estado, entre otras situaciones, pero que, en lugar de intimidar, han permitido al docente ser la esperanza de las comunidades campesinas y, a su vez, les ha significado la transformación de su práctica pedagógica desde la adversidad. 
Palabras clave: medio rural, narrativas, ciencias de la educación, formación de docentes, conflicto armado.

\begin{abstract}
:
Education in the rural context is determined by historical, political, social, economic and cultural situations which, despite its enormous importance for the most vulnerable population in Colombia, has long been invisible, marginalized, impoverished and estranged from academic and research discourses. This reflective article is an approach to the stories of rural teachers of the department of Antioquia based on the autobiographical narratives made in
\end{abstract}

2018 at the Pedagogical Thought Center, seeking to make visible the teaching work carried out in the rural area of the Northeast of Antioquia; where historically problems have emerged as a result of the armed conflict, violence, the abandonment of the State, among other situations; but which, instead of intimidating, have allowed teachers to be the hope of the peasant communities, and in turn, it has meant the transformation of their pedagogical practice from adversity.

Keywords: rural environment, narratives, Education Sciences, teacher training, armed conflict.

\section{Introducción}

El Centro de Pensamiento Pedagógico es una iniciativa de la Secretaría de Educación de Antioquia en alianza con distintas universidades de gran prestigio del departamento, como la Universidad de Antioquia, la Universidad EAFIT, la Universidad Pontificia Bolivariana, la Universidad de Medellín, el Tecnológico de Antioquia, Institución Universitaria, entre otras.

El Centro busca promover la investigación en relación con la cultura escolar y la profesionalidad docente. A esta iniciativa se sumaron maestras y maestros de diversos municipios del departamento desde distintas subregiones, quienes, a partir de la experiencia viva de la escuela, reflexionan, cuentan, narran sus trayectorias pedagógicas y personales para fortalecer la investigación académica; además de establecer puentes entre academia-escuela, así como entre los mismos docentes, con el propósito de valorar, visibilizar y repensar la escuela en todos los contextos del territorio.

Esta propuesta es de gran relevancia para la investigación educativa de Antioquia, puesto que pocas veces se establece un nexo directo entre el investigador y su objeto de estudio. En este caso, las culturas escolares y profesionalidades docentes son analizadas también desde la mirada de las maestras y maestros, que a la vez son sujeto de estudio, difuminándose la frontera entre el sujeto-objeto investigador y testigo de los hechos.

El esfuerzo por vincular la postura académica con las reflexiones docentes, que se expresan a partir de las cotidianidades de la escuela por medio de sus 
narrativas, instala a maestras y maestros en el campo de investigación y, a la vez, propicia la autorreflexión, lo que conduce al vínculo estrecho con su práctica y a empatizar con otras formas de ser y hacer la docencia. En este sentido, la reinvención se presenta como una bandera para la transformación de la escuela, que emerge desde las propias prácticas pedagógicas y que, de la mano con la investigación académica, permite pensar políticas públicas educativas que cuenten con el reconocimiento de los espacios y de las experiencias situadas para el mejoramiento de los procesos educativos en los diversos contextos que componen la escuela.

Desde la subregión del nordeste, la participación se dio con maestras y maestros de los municipios de Yolombó, Cisneros, Santo Domingo y San Roque, de distintos niveles de enseñanza y espacios, tanto urbanos como rurales. En la subregión, las miradas recogidas en los encuentros del Centro de Pensamiento permitieron sistematizar experiencias pedagógicas exitosas e identificar rasgos de la cultura escolar regional de las profesionalidades docentes; igualmente, expresar las particularidades, diferencias y matices de la educación en entornos urbanos y rurales, de los obstáculos o factores de riesgo que ponen en jaque los procesos educativos, pero también de las experiencias resilientes que emergen en la dificultad.

Así, este artículo nace como resultado de las consideraciones realizadas en los encuentros de la subregión nordeste en el marco del Centro de Pensamiento a partir de las narrativas compartidas por maestras y maestros de las diferentes instituciones educativas convocadas, con el deseo no solo de develar y compartir las voces desde la ruralidad frente al diario vivir del docente, sino también reflexionar la práctica pedagógica en contextos adversos, marcados por historias de conflicto, violencia y ausencia del Estado, emergidos como experiencias constantes en los relatos escritos y contados. Los encuentros, a su vez, permitieron evidenciar que las narrativas pedagógicas son un dispositivo potente que permite profundizar en el ser y en el hacer, como herramienta metodológica y teórica, y también de reflexión colectiva y acercamiento directo al sujeto y a su práctica.

Este texto presenta en primera instancia el marco metodológico utilizado que permitió el análisis de las narrativas; en segundo lugar, contextualiza y analiza los aspectos en los que las y los docentes desarrollan su práctica desde contextos rurales adversos o en conflicto; $y$, por último, plantea nuevas discusiones que surgen y dan pie a reflexiones en relación con la profesionalidad docente en la ruralidad.

\section{Navegando entre narrativas pedagógicas}

Este artículo reflexivo se construye a partir de la metodología de análisis documental o análisis de contenido de una serie de narrativas autobiográficas de maestras y maestros del nordeste del departamento de Antioquia, quienes dejan percibir en 
sus trayectorias profesionales, una serie de elementos, los cuales, en su conjunto, hacen posible un acercamiento a los territorios, en este caso, a las comunidades rurales y, a su vez, a las sensaciones, temores y oportunidades que hacen posible la transformación de la práctica pedagógica en medio de las dificultades en las que se desempeña la labor docente.

El enfoque metodológico de análisis externo permite que los investigadores realicen una lectura de las narrativas ubicando cada una en su contexto, es decir, en los momentos, tiempos, situaciones y lugares que dieron origen a la historia narrada, favoreciendo así una explicación mucho más cercana a la realidad. De esta manera, se constituyen ejes fundamentales para la interpretación de los hechos y para el estudio de los factores sociales, políticos, económicos, culturales, etc., que se entretejen en el discurso escrito a raíz de las experiencias vividas en diferentes comunidades rurales de la subregión, dándole un sentido al mensaje y reconociendo la trascendencia que puede tener para la academia, para otros investigadores o en el contexto mismo de donde surge el análisis (López, 2002).

Las narrativas desde una mirada global son textos que se enriquecen a partir de las emociones, las experiencias, los sucesos trascendentales que marcan no solo la vida profesional, sino también la vida personal de cada uno de los docentes que emplean este recurso para descubrir en su trayectoria su propia identidad. Así, el método de análisis de contenido, de acuerdo con López (2002), “[...] no es el estilo del texto lo que se pretende analizar, sino las ideas expresadas en él, siendo el significado de las palabras, temas o frases lo que intenta cuantificarse" (p. 173). En este sentido, al realizar la lectura de todas las narrativas de las maestras y maestros del nordeste antioqueño, se extraen aquellos fragmentos de texto en donde se logra evidenciar la complejidad del ejercicio de la labor docente a causa de la violencia, el conflicto armado, el abandono del Estado, etc. en el medio rural, y cómo esas problemáticas no terminan en un abatimiento, sino que sirve como impulso para construir, desde las adversidades, prácticas educativas que hacen posible una forma de ver y vivir el mundo para los niños y niñas al interior de la escuela, transformando poco a poco la realidad de las comunidades rurales desde la pedagogía.

\section{El docente rural en medio del conflicto: su voz, la voz de la esperanza}

La Colombia histórica tiene una identidad propia, determinada por un conflicto armado que ha desangrado los territorios rurales, dejando solo un mundo invisible, marginado, empobrecido y en un abandono sombrío (UNESCO, 2015). Los actores y objetivos del conflicto son diversos. En el medio se encuentra una sociedad que a razón del miedo ha sabido callar. En tal realidad emergen los rostros de los docentes rurales que construyen nación sobre la desesperanza y, en muchos casos en un 
total abandono del Estado, donde la escuela se presenta como el único territorio que representa la institucionalidad y, a la vez, un espacio neutral en el que las comunidades buscan refugio y atención no solo educativa, sino también psicológica y social. Ramírez (2019) realiza un análisis sobre las trayectorias profesionales de las maestras y maestros del nordeste antioqueño, argumentando que:

Los discursos y las prácticas pedagógicas de los maestros de la región dan cuenta, en su mayoría, de profesionales conscientes de las condiciones geográficas de difícil acceso a la escuela, la escasez de recursos educativos, las limitaciones de infraestructura escolar, la soledad [...] el silencio estatal, los conflictos militares políticos, la historia local de violencia [...] (p. 223).

Es evidente que la trayectoria profesional de las y los docentes, sus historias de vida y su práctica pedagógica se desarrollan en contextos empobrecidos, sin presencia estatal y desestructurados socialmente. Esos docentes, ajenos a su realidad, asumiendo el desarraigo, la separación de su familia y de la cultura en la que crecieron, responden a los retos que suponen dichas dificultades. Así lo manifiesta Valencia (2019), una docente que ha de enfrentarse al trasegar y la movilización dentro del mismo territorio, aunque en distintas comunidades:

Después de un año y medio, a través de un traslado municipal, pasé a otro corregimiento del mismo municipio. [...] En varias ocasiones escuchaba muchos disparos y luego todo quedaba en completo silencio, un silencio que se hacía terrible, para luego al amanecer, encontrarnos con el llanto de los niños y familias por la pérdida de sus seres queridos (p. 267).

El sentir de la maestra y el maestro en un territorio desconocido que intenta adoptar como propio, se desarrolla con tal fuerza, que aquello que le sucede al otro, lo vive con el mismo dolor. La realidad de la educación rural es que el conflicto armado y la violencia penetran hasta las relaciones sociales entre las personas que habitan un espacio geográfico común y, a su vez, determinan una dinámica que se despliega al interior y al exterior de la escuela (Bautista y Gonzáles, 2019, p. 13). Aunado a esto, el docente, al ser productor de significados y un agente socializador, quien da sentido a los espacios comunitarios, se convierte en un objetivo de guerra, desde un accionar psicológico, que busca intimidar desde el dominio de su rol a las comunidades (Lizarralde, 2003, p. 83). Todo esto es posible sentirlo y casi que vivirlo, al leer las palabras de Toro (2019), un docente que debió enfrentarse a la tragedia en medio de un momento que se suponía era para el goce pleno de los niños, niñas y adolescentes 
quienes se encontraban en la escuela, tratando de construir desde el conocimiento una historia propia, alejada de un sufrimiento que, para entonces, parecía no tener fin:

Pero el momento más impactante de mi carrera docente, fue el día que, estando en plena clase, un viernes de agosto del 2000, tipo 11:20 a.m., cuando cinco estudiantes ven pasar a sus padres amarrados de manos, unos a otros, custodiados por un grupo fuertemente armado. [...] Las lágrimas discurrían por nuestras mejillas cuando alcanzamos a escuchar a unos escasos ciento cincuenta metros de la escuela, en la carretera, los disparos, los tiros de gracia. “iLos mataron!”, dijo la hija mayor de uno de ellos. [...] iQué escena tan desgarradora! El llanto incontrolable de aquellos huérfanos (Toro, 2019, pp. 286-287).

Pese a los horrores del conflicto, las maestras y maestros son las voces de la esperanza en los contextos más adversos. La escuela se presenta no solo como un escenario de aprendizaje, sino como un refugio y una plataforma para vincularse a otras realidades y mundos posibles. La creación, la movilización comunitaria, el trabajo en equipo, la vinculación de los padres y madres de familia a la escuela, se convierten en las prácticas más comunes en los lugares más golpeados por la violencia y la pobreza, donde lo que se reclama más allá de las letras y los números, es la vida.

Una de las tareas del educador, [...] a través del análisis político serio y correcto, es descubrir las posibilidades — cualesquiera que sean los obstáculos para la esperanza, sin la cual poco podemos hacer porque difícilmente luchamos, y cuando luchamos como desesperanzados o desesperados es la nuestra una lucha suicida, un cuerpo a cuerpo puramente vengativo (Freire, 2002, p. 25).

La reinvención de las historias que han sido contadas y que hacen parte de la cultura de las comunidades campesinas, la verdadera hazaña que implica casi una revolución pedagógica, la inmersión en los espacios extraescolares y el compromiso social, económico y político que asume el docente, es quizá la forma de construirse a sí mismo, enfrentándose a responsabilidades que no debería asumir, que le son ajenas, que no hacen parte de la función que en teoría debe desempeñar. Empero, resulta imposible desconocer lo que pasa alrededor de la escuela, debido a las profundas secuelas que deja en las relaciones sociales, en las dificultades para enseñar a estudiantes que al parecer no pueden aprender, porque sus mentes están allá, en sus casas, en donde el hambre, el miedo, la orfandad o la miseria son la única forma que conocen de vida. 
En estas dinámicas, el docente desempeña en diversos territorios, en la ruralidad, sobre todo, el papel de mediador de conflictos, la voz del Estado ausente, que le ha puesto como un sujeto de amenaza y desplazamiento, o en el menor de los casos, sujeto de crítica moral y de transgresor de la tradición, pues su lucha se da, en muchas ocasiones, contra la misma resistencia de las comunidades que no son capaces de afrontar el entusiasmo de alguien externo que desconoce aquel mundo silencioso.

No obstante, el docente no se acobarda ante el sonido del fusil que hace eco entre los valles y montañas, ante el ataque de sus contradictores o los problemas de sus estudiantes para aprender un currículo que carece de sentido, por eso, la única forma posible de desvirtuar el mar de dificultades es la búsqueda para movilizar recursos, aunando esfuerzos de cooperación en la comunidad educativa, fortaleciendo lazos solidarios y de reciprocidad, construyendo proyectos, mejorando su práctica, ingeniando métodos que se alineen con los intereses y las necesidades del contexto. Esta labor, a veces tan desprestigiada, termina siendo, como lo manifiesta Franco (2019) en su narrativa, una constructora que edifica sobre cenizas y escombros un futuro diferente, que poco a poco se va forjando a raíz del esfuerzo, la cadencia, la voluntad y el espíritu de lucha que ha de caracterizar a la maestra y el maestro rural:

Para contrarrestar los episodios de violencia en la vereda, iniciamos con el apoyo de la administración municipal, la reconstrucción del tejido social a través de encuentros deportivos, culturales y religiosos; de este modo se minimizan los hechos pasados y entramos en una nueva etapa de vida escolar a pesar de ya no contar con varios de mis estudiantes en el aula de clase (p. 234).

El docente es el llamado a estar de frente a la realidad social de su contexto, es el que cotidianamente la vive, la interpreta y el que debe procurar, de ser necesario, la conciencia al cambio. Sin embargo, en contraste con la voz de las maestras y maestros y sus trayectorias biográficas narrativas, ha sido poco documentada la realidad de la educación rural, la cual podría servir como sustento académico a investigadores y universidades para que, a partir del acercamiento al territorio desde la literatura, se generen nuevas propuestas que favorezcan el desarrollo social de aquellos espacios en donde aún hay huellas visibles de un pasado que permanece en la memoria de campesinos, indígenas, afros y mestizos, quienes encarnan la soledad y el horror, y quienes luchan por un futuro diferente. 


\section{Transformación de la práctica pedagógica desde la adversidad}

La praxis científico-pedagógica del docente y la construcción del saber tienen un origen determinado por el “[...] encuentro de saberes y prácticas científicas con las cotidianas, vivenciales y laborales” (Runge y Murillo, 2018, p. 6). La profesión docente, más que otras quizá, está determinada por el juego de las subjetividades. El docente dotado de un conocimiento que desea replicar, encarna también un sujeto provisto de emocionalidad, preferencias, opiniones, en donde su historia de vida y experiencia es un constante insumo de saber, aprendizaje y, a la vez, su profesión misma, le permite adquirir, cambiar y repensarse al mismo tiempo que ejerce su labor. Por tanto, la trayectoria profesional marcada por el conflicto armado y la violencia, le exigen a la maestra y maestro cambios en su propia forma de ver y pensar la educación. Valencia (2019) deja en evidencia esa fuerza que emerge desde el interior, que recoge el paso por la academia y se mezcla con la necesidad de autoformarse a razón de las experiencias, para situarse en un tiempo y en un espacio, decidida a cambiar su entorno, pero, sobre todo, a cambiarse a sí misma:

No había vuelta atrás, mis miedos y mi inseguridad tenían que desaparecer, por ello, no dejaba que mi trabajo dejara de ser el mejor. Empecé por transformar mis prácticas pedagógicas a través del desarrollo de pequeños proyectos de interés de los estudiantes, aunque con recelo por los estudiantes que tenía en el aula, a los que sentía en muchas ocasiones como una amenaza (p. 268).

La ruralidad es un microsistema dentro de lo que es la educación en Colombia, lo que sucede en la Escuela Rural marca significativamente la práctica pedagógica de los docentes. De acuerdo con Brumat (2011): “[...] los maestros construyen su práctica más allá del aula, más allá de una dimensión estrictamente pedagógico-didáctica. En la práctica cotidiana van aprendiendo 'cosas que no les enseñaron en el magisterio"” (p. 6).

A pesar de esto, el desarrollo de la práctica pedagógica en el campo colombiano debe sortear diversas adversidades, esto lo deja entrever la historia que cuenta Duque (2019), una maestra que entiende la necesidad de enfrentarse a numerosos retos y solucionar problemas inesperados y es, en ese sistema de movilización y resignificación, que se genera una transformación real de lo que se hace al interior de las aulas:

Asumir los retos que me plantearon las anteriores dificultades, no me llevaron a pedir traslado o renunciar, en cambio inicié mi comprensión sobre la importancia de la educación, la lucha 
Narrativas sobre el saber pedagógico, entre el acierto y la adversidad: reflexiones desde la educación rural en el nordeste de Antioquia

constante, el amor en el acto de formar seres humanos útiles a la sociedad, promoviendo transformaciones y cambios en las prácticas culturales y educativas (p. 299).

La construcción de las realidades en los esquemas representativos del sistema educativo colombiano y, en general, de las prácticas pedagógicas y los modelos que intentan establecer un vínculo entre la escuela y el contexto, las necesidades de transformación a la luz del desarrollo social y político y las características de los niños y niñas que se desean formar, termina siendo absorbida por la historia vivida y vuelta a vivir en un movimiento cíclico sumido en las imposibilidades de abstracción frente a los mismos paradigmas que, aunque insuficientes, son los que parecen ser funcionales. En este sentido, una sociedad que construye su dinámica a raíz de los mismos momentos y situaciones contra los que lucha, se va a educar para perpetuarse a sí misma.

El docente es quien tiene la posibilidad desde su academia, identidad profesional, convicciones, creencias, pensamientos y prácticas educativas, de generar un cambio real, no necesariamente en función a la transformación de la realidad como respuesta al colosal mundo plagado de imaginarios, sino un desarrollo de procesos que no replique su propia formación, que no replique lo que se ha instaurado en la conciencia social colectiva, en el discurso, en la realidad histórica y que no se entumezca en el yermo silencio de los que no han tenido voz.

El cambio no exige una inmensa maquinaria desbordada de voluntad, exige, ciertamente, la predisposición del docente hacia un mundo diferente, esto significa que, su propia voz, sus gestos y sus enseñanzas, se concreten no como el refuerzo de la cultura existente, sino como impulso de una pedagogía moderna, una pedagogía liberadora, una pedagogía humanizada, una pedagogía centrada en la evolución del ser, del saber, de la cosmovisión de una sociedad cansada de vivir en medio de la adversidad, y es ahí, en donde el docente se torna en la fortaleza y voluntad de hacer creer y de hacer posible un verdadero cambio motivado por la escuela. El docente Flórez (2019) narra cómo, desde la pedagogía, logró convertir un problema comunitario en una fortaleza, la cual termina beneficiando en gran medida, a los niños y niñas, quienes se podrán formar en un ambiente más sano:

La comunidad se encontraba dividida por problemas personales entre familias y si lograba cambiar algo, eso sería convertirme en un gran líder y educador de la comunidad. [...] Gracias a un proyecto que realicé llamado: Quiero a mi comunidad, se empezó a realizar el cambio [...] (Flórez, 2019, p. 328). 
La transformación de la identidad y la trayectoria profesional, reflejada en propuestas pedagógicas alternativas, que se promueven como soluciones a los problemas con los que se encuentran los docentes en las comunidades rurales donde desarrollan su labor, demuestran la importancia que tiene para las maestras y maestros transitar por la ruralidad, por lo que, pese al infortunio, enseñar en las escuelas más distantes no termina siendo una completa frustración, ni siquiera un problema o un desafío profesional que hace desfallecer. En las narrativas se logra observar que la primera opción no es huir, todo lo contrario, el campo colombiano se convierte en una oportunidad de ser más fuerte, de aprender sobre una pedagogía inexistente, una pedagogía autóctona, que debe vivirse y sentirse para comprenderse, por lo que es necesario afirmar que la Escuela Rural es el laboratorio de experiencias que, aunque cargadas de conflictos y temores, permite la construcción de un saber pedagógico, entre el acierto y la adversidad. Para Sosa (2019), el hecho de estar demasiado lejos de su hogar, no le impedía ver y sentir el hermoso paisaje en donde quedaba incrustada, aparentemente, en medio de la nada, la escuela en donde empezaría a laborar:

[1]a vía a la escuela era solitaria y poco transitada, no había casas a orilla de la carretera [...] la zona no contaba con energía eléctrica, la región era montañosa, las casas distantes unas de otras, el paisaje era hermoso, el trinar de las aves alegraban los amaneceres y el croar de las ranas, las luciérnagas con su luz, el cantar de los grillos y las chicharras, acompañaban las noches, el olor de las flores de heliotropo impregnaban el ambiente, era un lugar fresco y tranquilo (pp. 275-276).

El saber científico, las teorías psicopedagógicas, la experiencia, el tiempo dedicado a la formación, la academia y la vida misma, dejan de tener sentido ante las situaciones que surgen en la ruralidad, la violencia, sobre todo, que aún es un fantasma vivo que ronda las cordilleras, las selvas, los ríos y veredas colombianas, le arrebata a maestras y maestros una visión sesgada de lo que es enseñar, debido a que el contenido curricular y aquellos escenarios de tranquilidad y paz tantas veces dibujados en los libros, en el débil paso por la universidad, en las ensoñaciones quiméricas que planteaban una escuela alejada de todos los problemas sociales del país, nada tienen que ver con las lágrimas, el miedo, la soledad, la muerte que se mira de frente, que aunque le pertenece a aquellos niños a quienes se desean formar, se encarna de manera personal, porque el sufrimiento de los estudiantes se convierte en el sufrimiento del docente, que solo le queda la tarea de consolar, sin tener quien lo consuele, y crear, desde el vacío y la nada, la manera de hacer que todo siga en pie, su curso natural, que la escuela continúe siendo la escuela y que la educación pueda brindar lo que el conflicto le ha arrebatado a lo largo de muchos años a la población 
pobre, dispersa, vulnerable y desconocida de esta tierra que parece no dolerle sus hijos, de ahí su importancia, pues en su ser está puesta la esperanza de que todo puede cambiar, que cualquier futuro puede ser mejor.

\section{En discusión: reivindicar la labor docente en el contexto rural}

La educación rural requiere de una mirada directa por parte de todos los actores involucrados en el sistema educativo nacional, con la intención de visibilizar la importancia que tiene en la trayectoria profesional y en la práctica pedagógica de las maestras y maestros. Esa caracterización de lo que poco se discute, poco se investiga y poco se publica desde la academia, se debe convertir en el eje fundamental de transformación, de cambio, de movilización hacia prácticas diferenciadas, que generen una nueva visión de lo que es la enseñanza en el campo, para que se promuevan propuestas que puedan ser incluidas en los programas de licenciatura de todas las universidades del país. Quizá, en el olvido intencionado contra el docente rural y proveniente del docente mismo, surja esa razón para dejar la queja, abandonar el miedo al otro, sacudirse del trámite burocrático, y empezar a caminar en torno a lo imaginado, porque en las ideas es de donde provienen las álgidas voluntades que contribuyen al cambio.

Teniendo en cuenta que a pesar del acuerdo de paz con uno de los grupos armados que más presencia tenía en el campo colombiano, aún se mantienen otros actores del conflicto que con su accionar afectan la vida y dignidad de las poblaciones rurales, y que en tales contextos los docentes desempeñan su labor, siendo tal vez la única presencia estatal de estas comunidades, la validación del saber pedagógico y la teoría educativa en estos contextos adversos cobra mayor relevancia porque le exige al docente la formulación de propuestas particulares y contextualizadas para generar prácticas educativas que trasciendan el aula y se reflejen en la necesidad de los niños y niñas y en las familias, quienes son el motivo por el cual se busca construir país sin importar la cantidad de dificultades que deban ser sorteadas.

La cultura y las cotidianidades determinan en gran medida, los verdaderos cambios en la profesionalización docente. Las narrativas, que reflejan la trayectoria, las emociones, los sentimientos, las percepciones y los pensamientos de los y las maestras hablan de prácticas transformadoras y se convierten en insumos potentes para el reconocimiento y socialización de experiencias que contribuyan a enriquecer políticas públicas educativas enfocadas en la educación rural. 


\section{El heroico acto de enseñar en la ruralidad colombiana, a modo de conclusiones}

- El accionar del docente trasciende el escenario de la escuela. En la ruralidad las maestras y maestros son permeados por el contexto que los acompaña y sus prácticas pedagógicas se convierten en motor de transformación que parte desde el aula hacia la comunidad.

- La ruralidad en Colombia, marcada por una historia de conflicto armado, convoca a la escuela a pensarse su papel activo para la construcción de una sociedad más democrática, determinada por la búsqueda de la protección de los derechos humanos y la disminución de las brechas de la desigualdad en el campo.

- Uno de los mayores obstáculos que deben afrontar los docentes rurales en Colombia es la poca presencia y acompañamiento que brinda el Estado. La maestra y el maestro son testigos de primera mano de las condiciones adversas de las poblaciones rurales en nuestro país. Carreteras inexistentes, servicios públicos deficitarios, infraestructura inadecuada y presencia de actores armados ilegales, entre otros, corresponden a las dificultades que afrontan los docentes en diversos lugares de la ruralidad.

- Las maestras y maestros constituyen la historia viva de la educación en Colombia. Sus narrativas son fuente de las diversas prácticas pedagógicas, cotidianidades, emociones y trayectos profesionales que aportan significados y marcan rutas para el reconocimiento de la labor docente, aportando insumos para el desarrollo de políticas públicas en los territorios.

\section{Referencias}

Bautista, M., y Gonzáles, G. (2019). Docencia rural en Colombia: educar para la paz en medio del conflicto armado. Fundación Compartir.

Brumat, M. (2011). Maestros rurales: condiciones de trabajo, formación docente y práctica cotidiana. Revista Iberoamericana de Educación, 55(4), 1-10.

Duque, V. (2019). Recuerdos memorables que dejan huella para siempre en un alma sencilla y sensible. En Relatos de experiencias maestras. Tomo 1. Gobernación de Antioquia.

Flórez, D. (2019). La extraordinaria vida de un maestro. En Relatos de experiencias maestras. Tomo 1. Gobernación de Antioquia.

Franco, B. (2019). Abriendo caminos. En Relatos de experiencias maestras. Tomo 1. Gobernación de Antioquia. 
Freire, P. (2002). Pedagogía de la esperanza. Un encuentro con la pedagogía del oprimido. Siglo veintiuno.

Lizarralde, M. (2003). Maestros en zonas de conflicto. Revista Latinoamericana de Ciencias Sociales, 2, 79-114.

López, F. (2002). El análisis de contenido como método de investigación. Revista de Educación, 21(4), 167-179.

Ramírez, P. (2019). Compartiendo saberes y significados pedagógicos. Relatos de experiencias maestras. Tomo 1. Gobernación de Antioquia.

Runge, A., y Murillo, G. (2018). Profesión Maestro y Cultura Escolar: Relatos de experiencia. Revista Brasileira de Pesquisa (Auto)Biográfica, 3(8), 397-415.

Sosa, E. (2019). Dieciocho años de aprendizaje en la educación. La docencia. En Relatos de experiencias maestras. Tomo 1. Medellín, Colombia: Gobernación de Antioquia.

Toro, J. (2019). La docencia. En Relatos de experiencias maestras. Tomo 1. Gobernación de Antioquia.

UNESCO. (2015). Informe de resultados: antecedentes iniciales. Tercer Estudio Regional Comparativo y Explicativo. Organización de las Naciones Unidas para la Educación, La Ciencia y la Cultura, UNESCO.

Valencia, M. (2019). No nací maestra. En Relatos de experiencias maestras. Tomo 1. Gobernación de Antioquia. 